\section{Mortalidade infantil no contexto da ruralidade brasileira: uma proposta para a superação da invisibilidade epidemiológica e demográfica}

\author{
Infant mortality in the Brazilian countryside: \\ a proposal to overcome epidemiological and \\ demographic invisibility
}

\begin{abstract}
Mortalidad infantil en el contexto de la ruralidad brasileña: una propuesta para la superación de la invisibilidad epidemiológica y demográfica
\end{abstract}

\section{Resumo}

O estudo teve como objetivo comparar duas propostas de ruralidade, do Instituto Brasileiro de Geografia e Estatística (IBGE) e do Banco Mundial, respondendo qual delas melhor adéqua-se à realidade no território e conhecer o perfil da mortalidade infantil nos municípios rurais da Paraíba, Brasil, segundo o critério de ruralidade melhor avaliado. Trata-se de um estudo epidemiológico observacional, realizado no Estado da Paraíba. O método de análise das tipologias rural/urbano baseou-se na mineração de dados, com base no algoritmo de associação Apriori. Para a análise do óbito infantil, utilizou-se a estatística descritiva. Considerou-se os dados do Sistema de Informações sobre Mortalidade do Ministério da Saúde do Brasil, no período de 2007 a 2016, e indicadores municipais do IBGE. A tipologia de ruralidade do Banco Mundial apresentou kappa $=0,337$ e a do $I B G E$ kappa $=0,616$. Entre os 223 municípios analisados, a tipologia do Banco Mundial classificou corretamente $130(65,66 \%)$ e a do IBGE 183 (82,06\%). O perfil epidemiológico predominante nos municípios rurais paraibanos foi sexo masculino $(57,4 \%)$, cor parda (61,1\%), idade entre 0 e 7 dias (52,4\%), com baixo peso ao nascer (44\%) e idade gestacional menor que 37 semanas (43,2\%). A causa básica da morte foi classificada como morte evitável por intervenções do Sistema Único de Saúde (65,2\%). A tipologia urbano/rural apresentada pelo IBGE classificou melhor os municípios paraibanos que a do Banco Mundial. Essa classificação possibilitou o estudo do perfil do óbito infantil nos municípios rurais, por meio do qual verificou-se, exceto pela escolaridade da mãe, similaridade com o perfil geral.

Mortalidade Infantil; Estatísticas Vitais; Saúde da População Rural;

Determinantes Sociais da Saúde; Equidade em Saúde
Rackynelly Alves Sarmento Soares 1

Ronei Marcos de Moraes 1

Rodrigo Pinheiro de Toledo Vianna 1

doi: 10.1590/0102-311X00068718

Correspondência

R. A. S. Soares

Av. João Cirilo da Silva 1700, casa 347, João Pessoa, PB

58046-005, Brasil.

rackynelly@gmail.com

1 Programa de Pós-graduação em Modelos de Decisão e Saúde, Universidade Federal da Paraíba, João Pessoa, Brasil. 
A mortalidade infantil é um indicador de saúde que revela a qualidade de vida e dos serviços de saúde prestados à população materno-infantil, sendo capaz de indicar os níveis de saúde, desenvolvimento social e econômico de determinada população 1,2,3. Destacando-se, por essas razões, como uma medida norteadora de políticas e ações em saúde 4.

A taxa de mortalidade infantil (TMI) vem declinando de forma expressiva em todo o mundo após 1990 5. Na região das Américas, a TMI reduziu de 17,9 mortes por mil nascidos vivos entre os anos de 2002 e 2005, para 13,6 por mil nascidos vivos entre os anos de 2010 e 2013 6. No Brasil, a TMI reduziu de 47 mortes por mil nascidos vivos em 1990 para 15,7 mortes por mil nascidos vivos em 20157 .

A mortalidade infantil é uma prioridade sanitária em pactos nacionais e internacionais, entre os quais destacam-se os Objetivos de Desenvolvimento do Milênio (ODM) para o ano de 2015 e mais tarde, os Objetivos do Desenvolvimento Sustentável (ODS) para o ano de 2030 3,6.

As desigualdades sociais do Brasil refletem diretamente na mortalidade infantil, conquanto este indicador esteja diminuindo gradualmente no país, as taxas ainda são muito elevadas nas regiões Nordeste e Norte 8. De forma a evitar que essa redução aconteça de forma homogênea, especialmente, entre grupos populacionais em situação de maior vulnerabilidade, como aqueles inseridos nos espaços rurais 9 .

Em função disso, ressalta-se a importância de políticas que contemplem as populações do campo, floresta e águas, também denominadas "população rural", que são todas as identidades rurais cujos modos de vida têm profunda relação com o primeiro setor, a exemplo dos camponeses, agricultores familiares, trabalhadores rurais assentados e acampados, comunidades de quilombos, populações que habitam ou usam reservas extrativistas, populações ribeirinhas, populações atingidas por barragens e outras comunidades tradicionais, conforme enumera a Portaria no 2.866, de 2 de dezembro de 201110.

Apesar de haver um considerável número de pesquisas relacionadas à mortalidade infantil, observa-se a escassez de estudos nos quais a mortalidade infantil contempla esses recortes populacionais, inseridos em espaços rurais. Tais estudos, entretanto, devem ser estimulados, visto que são fundamentais para a superação da "invisibilidade demográfica e epidemiológica" dessa população 9.

O Sistema de Informações sobre Mortalidade (SIM) não dispõe de uma variável que relacione o óbito às áreas rurais, situação que impede o cálculo direto da taxa de mortalidade infantil por área rural 11. O Programa das Nações Unidas para o Desenvolvimento (PNUD) 12 também identificou essa lacuna em relação ao SIM e ao próprio Censo Demográfico de 2000 do Instituto Brasileiro de Geografia e Estatística (IBGE; http://www.ibge.gov.br, acessado em 20/Ago/2013), evidenciando a impossibilidade de desagregação desses dados por situação de domicílio. Sendo essa uma possível explicação para a escassez de estudos sobre a mortalidade rural no Brasil.

Nesse sentido, estudar a mortalidade infantil na perspectiva da ruralidade deve colaborar com o entendimento da situação de saúde nos espaços rurais, favorecendo os processos de implantação, avaliação e monitoramento de políticas, programas e ações de saúde voltados para este recorte populacional.

Para além da temática da mortalidade, a utilização de uma tipologia adequada para a classificação rural/urbano na escala municipal possibilita estudos com dados de outros sistemas de informação em saúde, sendo fundamental, antes de tudo, uma definição de ruralidade consistente.

Na América Latina e Caribe, observa-se a tendência de subestimativa do tamanho e da importância das áreas rurais, bem como, da diversidade de critérios na definição oficial do rural 13 . O critério do tamanho populacional é usado pela Argentina, Bolívia e Venezuela. Somado a esse critério, Honduras e Cuba agregam a oferta de serviços. Chile considera, unicamente, a participação da população que vive da agricultura; já o Peru considera a aglomeração de propriedades. No Brasil, Colômbia, República Dominicana, Equador, El Salvador, Guatemala, Haiti e Paraguai adota-se uma divisão administrativa. Por fim, Costa Rica e Uruguai, além da divisão administrativa, consideram a oferta de serviços 13 .

A complexidade desses espaços, a relação dinâmica campo/cidade de interdependência e de interpenetração espacial não podem ser representadas pela dicotomia estática urbano-rural 14. No Brasil, a classificação territorial rural/urbano político-administrativa que define os limites oficiais dessas áreas é baseada em decisão legislativa municipal 15.

Entretanto, essa tipologia não consegue representar a heterogeneidade do rural brasileiro e, por isto, vem sendo objeto de vários estudos que sugerem critérios mais consistentes nessa definição 13,15. 
Por essa razão, este estudo se depara com duas definições de ruralidade. A primeira foi proposta pelo Banco Mundial 16 que apresenta graus de ruralidade considerando informações municipais acerca da densidade demográfica e da distância para as grandes cidades, segundo o tempo de viagem. Essa característica, em especial, é relevante para a discussão da mortalidade infantil porque afeta o acesso aos serviços de saúde. Segundo Unglert et al. 17 (p. 439), "o grau de acesso real aos serviços de saúde depende (...) da distância que se deve percorrer para obtê-los, do tempo que leva a viagem e do seu custo".

A segunda definição de ruralidade adotada foi publicada no último trimestre de 2017 pelo IBGE 15. Ela pretende considerar elementos contemporâneos, como "aumento das atividades não agrícolas, a mecanização, a intensificação da pluriatividade, a valorização da biodiversidade, a expansão do setor terciário e a intensificação de fluxos materiais e imateriais" 15 (p. 8). Considerou três critérios: população em áreas de ocupação densa, proporção da população em áreas de ocupação densa em relação à população total e localização; definindo cinco categorias rural/urbana para os municípios brasileiros: Urbano, Intermediário Adjacente, Intermediário Remoto, Rural Adjacente e Rural Remoto 15.

Serão comparadas as propostas de ruralidade do IBGE e do Banco Mundial, buscando identificar qual delas representa melhor o Estado da Paraíba e, em seguida, caracterizar o perfil da mortalidade infantil para os municípios rurais da Paraíba segundo o critério de ruralidade melhor avaliado.

\section{Materiais e métodos}

Trata-se de um estudo ecológico 18 utilizando série de óbitos de menores de um ano de idade, não fetais, ocorridos na Paraíba, no período de 2007 a 2016.

Para a caracterização dos óbitos infantis, as informações foram coletadas no SIM e incluídas as seguintes variáveis: data do óbito, sexo, raça/cor, idade da criança, município de residência da mãe, local de ocorrência do óbito, município de ocorrência do óbito, idade da mãe, escolaridade da mãe, gravidez, gestação, tipo de parto e peso ao nascer.

Os óbitos foram classificados segundo o critério de morte evitável por intervenções do Sistema Único de Saúde (SUS), baseando-se na classificação dos óbitos infantis em causas evitáveis (sim/não), da lista brasileira de mortes evitáveis ${ }^{19}$. Segundo esse critério, os óbitos evitáveis de recém-nascidos são aqueles reduzíveis pela adequada atenção à mulher na gestação, ao parto e ao recém-nascido 18 . Foram excluídos os óbitos infantis que não apresentaram o município de residência da mãe.

\section{Aplicação dos critérios de ruralidade municipal no Estado da Paraíba}

Para a comparação das tipologias rural/urbano, foram utilizados dados geográficos municipais no formato shapefile e dados do Censo Demográfico de 2010 (densidade demográfica).

Para a aplicação dos critérios de ruralidade do Banco Mundial - distância para cidades com pelo menos 100 mil habitantes (menos de 1 hora e mais de 1 hora) e densidade demográfica (até 20 pessoas por $\mathrm{km}^{2}$, até 50 pessoas por $\mathrm{km}^{2}$, até 100 pessoas por $\mathrm{km}^{2}$ e maior que 100 pessoas por $\mathrm{km}^{2}$ ) - foram usadas técnicas de geoprocessamento para espacialização em escala municipal desta tipologia. Inicialmente, procedeu-se à identificação das "grandes cidades".

Para o cálculo do tempo de viagem, em relação às grandes cidades, utilizou-se a distância em linha reta entre os municípios, considerando como ponto de partida/chegada o centroide do polígono de cada município paraibano que apresentou mais de 100 mil habitantes, segundo o Censo Demográfico de 2010. Em seguida, foram criados buffer zones de $60 \mathrm{~km}$ em torno desses centroides.

Considerou-se buffer de $60 \mathrm{~km}$ por ser essa a distância possível de alcançar em uma hora de viagem, adotando-se como velocidade máxima $60 \mathrm{~km} / \mathrm{h}$, tal como regulamenta o Código de Trânsito Brasileiro 20 em seu Capítulo III, Art. 61 e, portanto, mais compatível com as estradas localizadas nas áreas mais rurais.

Por fim, realizou-se uma consulta espacial considerando os buffers zones como critério para a categorização do tempo de viagem. Assim, o centroide do município que estava contido no raio do buffer zone foi categorizado como "tempo de viagem até 1 hora", e aquele que estava fora deste raio foi categorizado como "tempo de viagem maior que 1 hora" (Figura 1). 


\section{Figura 1}

Mapa dos municípios da Paraíba, Brasil, contidos e não contidos em buffer zones de 60km, segundo a distância para cidades com pelo menos 100 mil habitantes.
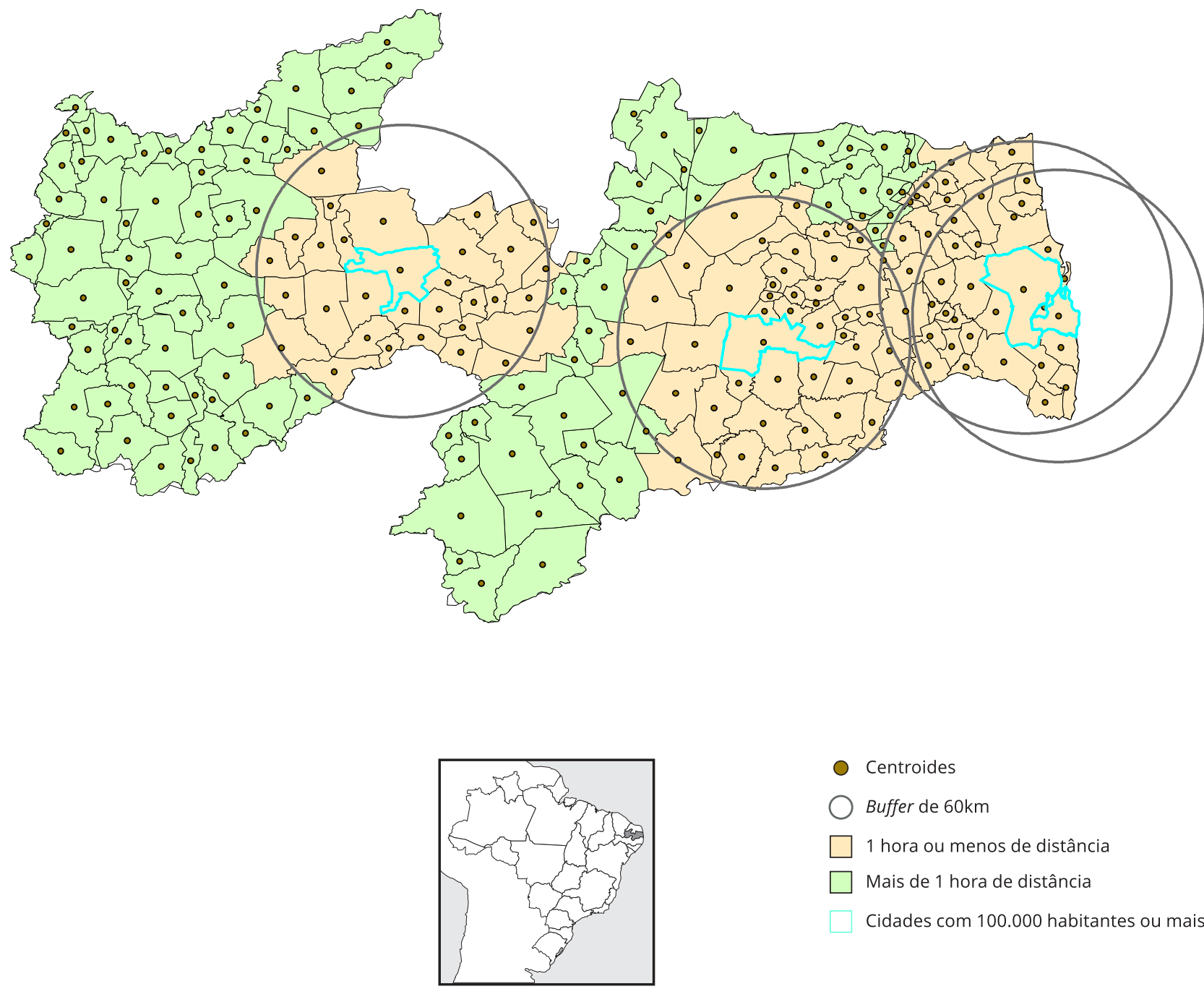

Centroides

Buffer de $60 \mathrm{~km}$

1 hora ou menos de distância

Mais de 1 hora de distância

Cidades com 100.000 habitantes ou mais

Em seguida, foram atribuídos aos municípios “pesos de ruralidade”, à luz de Sorokin et al. 21, que variaram de 2 a 6, os quais foram armazenados na variável "grau de ruralidade", de modo que quanto maior o número, maior a ruralidade do município.

A relação entre as duas tipologias considerou: município com grau de ruralidade 2 como sendo equivalente a município Urbano; com graus de ruralidade 3 e 4 equivalentes a Intermediário Adjacente; e com graus de ruralidade 5 e 6 equivalentes a Rural Adjacente. Com isso, os cinco níveis de ruralidade do método do Banco Mundial foram compatibilizados com os três níveis de ruralidade do método do IBGE existentes no Estado da Paraíba (nenhum município paraibano é classificado pelo IBGE como Rural Remoto ou Intermediário Remoto). 


\section{- Comparação das duas propostas - Banco Mundial vs. IBGE}

Para a comparação entre as tipologias do IBGE 15 e do Banco Mundial 16 foram utilizadas as variáveis: código do município, ruralidade_Banco_Mundial e ruralidade_IBGE. Também foram incluídos três indicadores de controle, relacionados com os espaços rurais 13,21,22 e que não foram usados como critério de classificação em nenhum dos métodos: (i) percentual dos ocupados no setor agropecuário (\%_AGRO $\geq 50 \%$, \%_AGRO < 50\%); (ii) percentual de população residente em situação de domicílio rural (\%_RURAL $\geq 50 \%$, \%_RURAL < 50\%), disponibilizados pelo Censo Demográfico de 2010; e (iii) Índice de Desenvolvimento Humano Municipal (IDHM baixo, IDHM médio e IDHM alto), disponibilizado pelo Atlas do Desenvolvimento Humano no Brasil (http://atlasbrasil.org.br/2013/). Essa base de dados municipal foi separada em dois conjuntos: concordantes e discordantes. O conjunto de concordantes foi composto pelos municípios classificados da mesma forma para as duas tipologias, sendo considerado como classificação correta.

A utilização do primeiro indicador encontra respaldo teórico na Sociologia, que destaca importantes diferenças entre os espaços urbanos e os rurais que são genéricos no espaço e, relativamente, constantes no tempo 21. Entre essas diferenças estão as atividades ocupacionais mais voltadas para o setor primário, no caso dos espaços rurais.

O segundo indicador, embora seja subdimensionado, tem extrema relevância para o estudo, visto que se espera uma população residente em área rural maior do que aquela declarada no Censo Demográfico de 2010, em que pese o fluxo migratório e todas as dinâmicas territoriais envolvidas 13 .

O terceiro indicador ressalta as desigualdades ainda presentes no território brasileiro, evidenciadas em vários estudos 12,22. De tal modo que, ao desagregar o IDHM por situação de domicílio, evidencia-se baixo IDHM nos espaços rurais, contrapondo-se ao IDHM dos espaços urbanos, que é considerado alto 12 .

Em seguida, foi criada a variável "comparação", que foi preenchida com a ruralidade considerada mais adequada à realidade paraibana, sendo composta pelo conjunto de concordantes e ainda pela classificação resultante das regras de associação aplicadas ao conjunto dos discordantes.

Valendo-se do conjunto de concordantes foram extraídas 10 regras de associação Apriori (disponível no software WEKA - https://www.cs.waikato.ac.nz/ml/weka/), baseadas nos três indicadores de controle (IDHM, \%_AGRO e \%_RURAL). A associação Apriori é considerada uma técnica de mineração de dados, de aprendizagem não supervisionada e que identifica padrões relevantes em um conjunto de dados. Esses padrões são estruturados na forma de regras de associação entre os valores dos atributos cujas principais medidas de qualidade são denominadas de confiança e suporte. Considerando $\mathrm{X}$ o conjunto de indicadores e $\mathrm{Y}$ a classificação do município, confiança refere-se ao número de registros que contém $\mathrm{X}$ e $\mathrm{Y}$ dividido pelo número de registros que contêm $\mathrm{X}$. E suporte refere-se ao número de registros que contêm $\mathrm{X}$ e $\mathrm{Y}$ dividido pelo número total de registros 23 .

Para fins deste estudo adotou-se o algoritmo de associação Apriori disponível no WEKA, com confiança 0,9; suporte 0,1; e número máximo de regras 10 , objetivando filtrar as melhores regras, com maior precisão e maior cobertura:

- Regra 1: se IDHM = baixo e \%_RURAL $\geq 50 \%$ então município = Rural Adjacente $($ lift = 1,32);

- Regra 2: Se \%_AGRO $\geq 50 \%$ então município = Rural Adjacente (lift = 1,32);

- Regra 3: Se \%_AGRO $\geq 50 \%$ e IDHM = baixo então município = Rural Adjacente $($ lift = 1,32);

- Regra 4: Se \%_AGRO $\leq 50 \%$ e IDHM = baixo e \%_RURAL $\geq 50 \%$ então município = Rural Adjacente $($ lift $=1,32)$;

- Regra 5: Se município = Urbano então \%_AGRO $\leq 50 \%($ lift = 1,32);

- Regra 6: Se \%_AGRO $\geq 50 \%$ e \%_RURAL $\geq 50 \%$ então município = Rural Adjacente $($ lift = 1,32);

- Regra 7: Se \%_RURAL $\leq 50 \%$ e município = Urbano então \%_AGRO $\leq 50 \%$ (lift = 1,32);

- Regra 8: Se \%_AGRO $\geq 50 \%$ e IDHM = baixo e \%_RURAL $\geq 50 \%$ então município = Rural Adjacente (lift = 1,32);

- Regra 9: Se \%_AGRO $\geq 50 \%$ e \%_RURAL $\leq 50 \%$ então município = Rural Adjacente $($ lift = 1,32);

- Regra 10: Se \%_RURAL $\geq 50 \%$ então município = Rural Adjacente $($ lift = 1,26). 
O pequeno número de municípios classificados como Intermediário Adjacente no conjunto de concordantes não permitiu a identificação de um padrão e, por isto, estes municípios não foram considerados na comparação. Foram construídas duas matrizes de confusão relacionando as tipologias do Banco Mundial e do IBGE com a variável "comparação", e a melhor tipologia foi aquela que apresentou melhor percentual de acerto e melhor coeficiente kappa 24 como resposta a esta comparação.

Este trabalho utilizou dados que estão sob domínio público, sendo dispensada da apreciação por Comitê de Ética em Pesquisa. Todos os princípios da Resolução no 466, de 12 de dezembro de 2012, do Conselho Nacional de Saúde, foram seguidos em todas as etapas da pesquisa.

\section{Resultados}

\section{Comparação entre os métodos Banco Mundial vs. IBGE}

Observa-se nas Figuras 2a e 2b que o Estado da Paraíba é majoritariamente Rural Adjacente, segundo as duas tipologias, sendo mais expressiva na tipologia do IBGE com 74,4\% e 46,6\% do Banco Mundial. Verifica-se, ainda, que a classificação dos municípios urbanos foi bastante semelhante nas duas tipologias, havendo pouca divergência. Marcadamente, a tipologia do Banco Mundial concentrou os municípios mais Urbanos e Intermediários Adjacentes nas mesorregiões da Zona da Mata e do Agreste, porção situada mais ao leste do estado.

Identificou-se que, dos 223 municípios paraibanos, 121 (54,26\%) obtiveram a mesma classificação pelas duas tipologias. Entre os municípios concordantes evidencia-se que apenas 11 foram classificados como Intermediários Adjacentes. Esse pequeno número de municípios que se destaca, ainda, pelo fato de serem localizados em área de transição entre urbano/rural, o que impossibilitou a identificação de um padrão pelo método de associação Apriori. Por essa razão, a comparação entre as duas tipologias considerou apenas os municípios Urbanos e Rurais, deixando de fora os Intermediários Adjacentes.

A tipologia do IBGE apresentou maior concordância com o padrão, apresentando maior percentual de classificações equivalentes e maior estatística kappa, sendo, portanto, adotada nas análises seguintes deste trabalho. O Banco Mundial apresentou kappa =0,337 e o IBGE apresentou kappa $=0,616$. Quanto ao percentual de acerto, o Banco Mundial obteve $130(58,3 \%)$ e o IBGE 183 $(82,06 \%)$ (Tabela 1).

\section{Perfil epidemiológico da mortalidade infantil na Paraíba (2007-2016)}

Do total de 586.375 nascidos vivos na Paraíba, no período do estudo, 8.609 morreram antes de completar um ano, resultando em uma TMI média de 14,66 por mil nascidos vivos. Em praticamente todo o período do estudo, observa-se a redução progressiva da TMI, que variou de 18,56 em 2006 para 11,60/mil nascidos vivos em 2015, representando uma redução de 37,5\%. Entretanto, em 2016 identificou-se o aumento do número de óbitos infantis e redução do número de nascidos vivos, implicando o aumento da TMI para 12,64/mil nascidos vivos e representando um aumento de $8,15 \% \mathrm{em}$ relação ao ano anterior.

Após aplicado o critério de exclusão, restaram 8.583 óbitos para a análise da distribuição em função da ruralidade. Entre esses óbitos, 2.627 (30,6\%) aconteceram em municípios Rurais Adjacentes (Tabela 2). Tal como verificado em todos os graus de ruralidade, os óbitos infantis foram majoritariamente do sexo masculino $(57,4 \%)$ nos municípios Rurais Adjacentes (Tabela 2).

Com relação à raça/cor, foram registrados $61,1 \%$ de óbitos de crianças de cor parda e 27,9\% de cor branca, nos municípios Rurais Adjacentes. As maiores frequências de óbitos infantis de cor parda ocorreram nos municípios Intermediários Adjacentes (67,9\%). Essa foi a única variável do estudo em que os municípios Urbanos apresentaram maior percentual de "ignorado" do que os Rurais Adjacentes (respectivamente, $11,4 \%$ e 9,2\%) (Tabela 2).

Quanto à idade da criança no momento do óbito, ressalta-se que a idade neonatal resultou em $70,7 \%$ dos óbitos, revelando ser este um dos grandes desafios da mortalidade infantil para os gestores de saúde, sobretudo para os municípios Intermediários Adjacentes que totalizaram $72,1 \%$. Os 


\section{Figura 2}

Mapa dos municípios da Paraíba, Brasil, segundo a tipologia rural/urbana.

2a) Tipologia do Banco Mundial

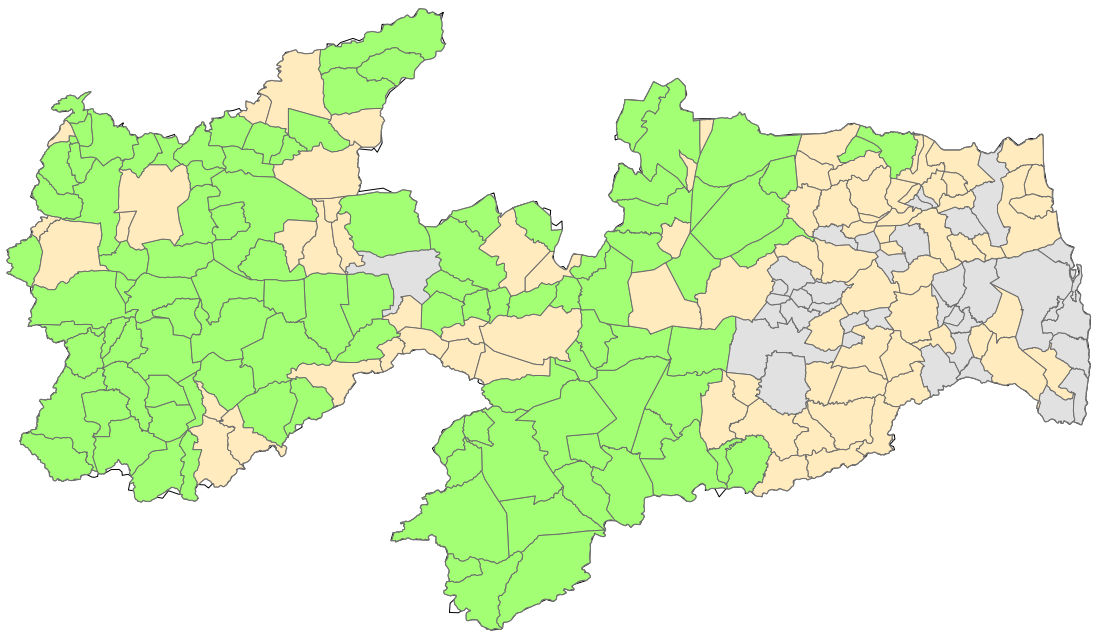

2b) Tipologia do IBGE
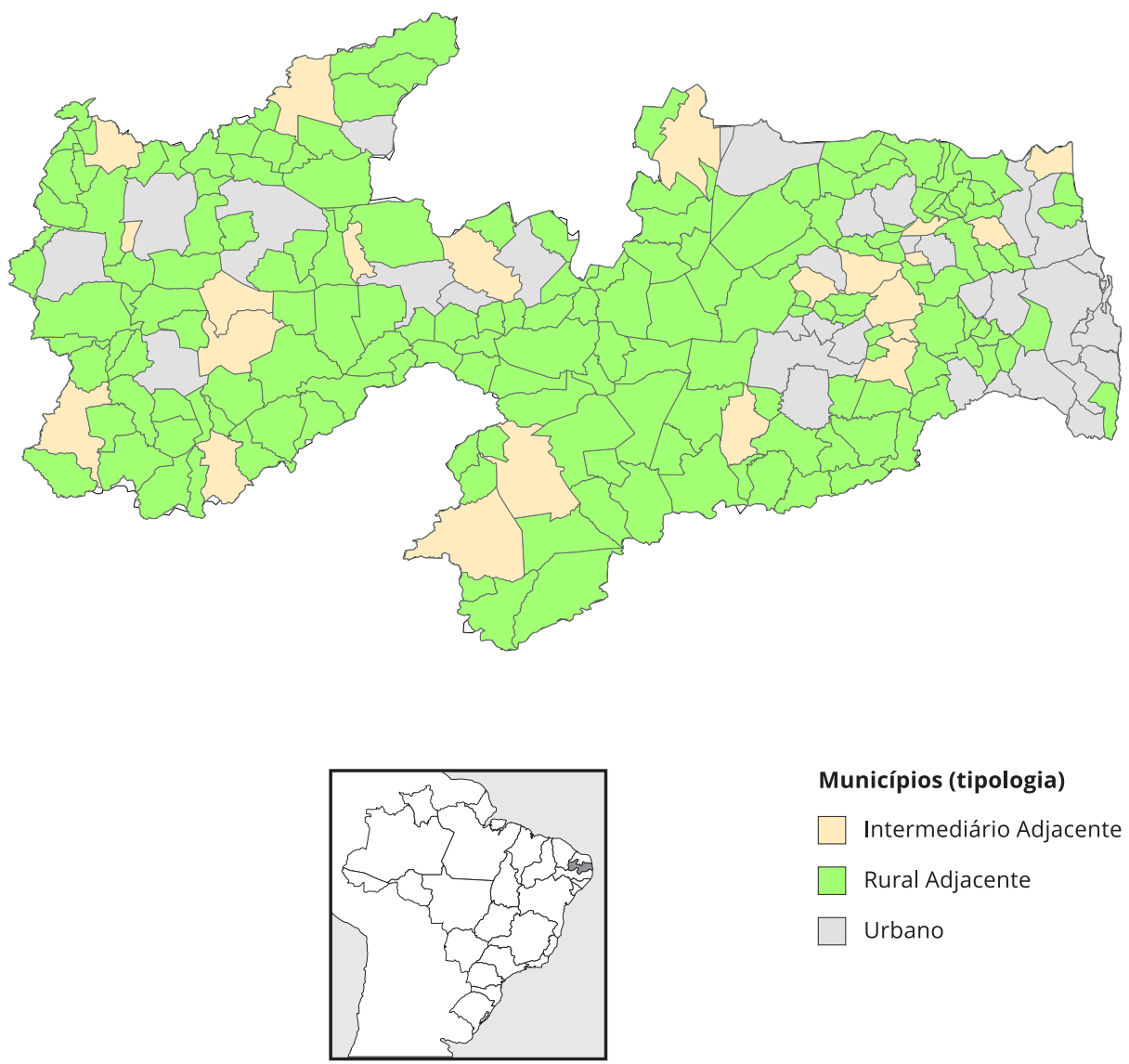

Municípios (tipologia)

Intermediário Adjacente

Rural Adjacente

Urbano 
Tabela 1

Matriz de confusão da classificação municipal segundo a tipologia rural/urbana em contraposição à variável "comparação".

\begin{tabular}{lccc}
\hline & Rural Adjacente & $\begin{array}{c}\text { Valor previsto } \\
\text { Intermediário Adjacente }\end{array}$ & Urbano \\
\hline Banco Mundial & $\mathbf{9 2}$ & 0 & 12 \\
$\quad$ Rural Adjacente & 41 & $\mathbf{1 1}$ & 29 \\
Intermediário Adjacente & 11 & 0 & $\mathbf{2 7}$ \\
Urbano & $\mathbf{1 4 0}$ & 0 & 26 \\
IBGE & 1 & $\mathbf{1 1}$ & 10 \\
Rural Adjacente & 3 & 0 & $\mathbf{3 2}$ \\
Intermediário Adjacente & & & \\
Urbano & &
\end{tabular}

IBGE: Instituto Brasileiro de Geografia e Estatística.

Fonte: elaboração própria, a partir de dados do Censo Demográfico de 2010 (IBGE. http://www.ibge.gov.br, acessado em 20/Ago/2013).

\section{Tabela 2}

Caracterização da mortalidade infantil (menos de 1 ano) na Paraíba, Brasil, segundo a tipologia rural/urbano municipal, 2007 a 2016.

\begin{tabular}{|c|c|c|c|c|c|c|c|c|}
\hline \multirow[t]{3}{*}{ Variável } & \multicolumn{6}{|c|}{ Tipologia } & \multicolumn{2}{|c|}{ Total $(\mathrm{N}=\mathbf{8 . 5 8 3})$} \\
\hline & \multicolumn{2}{|c|}{ Rural Adjacente } & \multicolumn{2}{|c|}{ Intermediário Adjacente } & \multicolumn{2}{|c|}{ Urbano } & \multirow[b]{2}{*}{$\mathrm{n}$} & \multirow[b]{2}{*}{$\%$} \\
\hline & $\mathbf{n}$ & $\%$ & $\mathbf{n}$ & $\%$ & $\mathbf{n}$ & $\%$ & & \\
\hline \multicolumn{9}{|l|}{ Sexo } \\
\hline Feminino & 1.107 & 42,1 & 367 & 42,8 & 2.198 & 43,5 & 3.672 & 42,8 \\
\hline Masculino & 1.507 & 57,4 & 489 & 57,0 & 2.875 & 56,4 & 4.871 & 56,8 \\
\hline Ignorado & 13 & 0,5 & 2 & 0,2 & 25 & 0,5 & 41 & 0,5 \\
\hline \multicolumn{9}{|l|}{ Raça/Cor } \\
\hline Branca & 733 & 27,9 & 202 & 23,5 & 1.398 & 27,4 & 2.333 & 27,2 \\
\hline Preta & 34 & 1,3 & 11 & 1,3 & 53 & 1,0 & 98 & 1,1 \\
\hline Amarela & 2 & 0,1 & 0 & 0,0 & 4 & 0,0 & 6 & 0,1 \\
\hline Parda & 1.605 & 61,1 & 583 & 67,9 & 3.055 & 59,9 & 5.243 & 61,1 \\
\hline Indígena & 12 & 0,5 & 1 & 0,1 & 8 & 0,2 & 21 & 0,2 \\
\hline Ignorado & 241 & 9,2 & 61 & 7,1 & 580 & 11,4 & 882 & 10,3 \\
\hline \multicolumn{9}{|l|}{ Idade da criança } \\
\hline Neonatal precoce & 1.376 & 52,4 & 466 & 54,3 & 2.728 & 53,5 & 4.570 & 53,2 \\
\hline Neonatal tardia & 411 & 15,6 & 153 & 17,8 & 940 & 18,4 & 1.504 & 17,5 \\
\hline Pós-neonatal & 840 & 32,0 & 239 & 27,9 & 1.430 & 28,1 & 2.509 & 29,2 \\
\hline \multicolumn{9}{|l|}{ Peso ao nascer (g) } \\
\hline$\geq 2.500$ & 782 & 29,8 & 236 & 27,5 & 1.164 & 22,8 & 2.182 & 25,4 \\
\hline$<2.500$ & 1.156 & 44,0 & 406 & 47,3 & 2.896 & 56,8 & 4.458 & 51,9 \\
\hline Ignorado & 689 & 26,2 & 216 & 25,2 & 1.038 & 20,4 & 1.943 & 22,6 \\
\hline \multicolumn{9}{|c|}{ Morte evitável por intervenções do SUS } \\
\hline Sim & 1.714 & 65,2 & 592 & 69,0 & 3.519 & 69,0 & 5.825 & 67,9 \\
\hline Não & 913 & 34,8 & 266 & 31,0 & 1.579 & 31,0 & 2.758 & 32,1 \\
\hline
\end{tabular}

(continua) 
Tabela 2 (continuação)

\begin{tabular}{|c|c|c|c|c|c|c|c|c|}
\hline \multirow[t]{3}{*}{ Variável } & \multicolumn{6}{|c|}{ Tipologia } & \multicolumn{2}{|c|}{ Total $(\mathbf{N}=8.583)$} \\
\hline & \multicolumn{2}{|c|}{ Rural Adjacente } & \multicolumn{2}{|c|}{ Intermediário Adjacente } & \multicolumn{2}{|c|}{ Urbano } & \multirow[b]{2}{*}{$\mathbf{n}$} & \multirow[b]{2}{*}{$\%$} \\
\hline & $\mathbf{n}$ & $\%$ & $\mathbf{n}$ & $\%$ & $\mathbf{n}$ & $\%$ & & \\
\hline \multicolumn{9}{|c|}{ Local da ocorrência do óbito } \\
\hline Hospital & 2.299 & 87,5 & 749 & 87,3 & 4.754 & 93,3 & 7.802 & 90,9 \\
\hline Domicílio & 209 & 8,0 & 58 & 6,8 & 260 & 5,1 & 527 & 6,1 \\
\hline Outros & 111 & 4,2 & 47 & 5,5 & 76 & 1,5 & 234 & 2,7 \\
\hline Ignorado & 8 & 0,3 & 4 & 0,5 & 8 & 0,2 & 20 & 0,2 \\
\hline \multicolumn{9}{|c|}{ Idade da mãe (anos) } \\
\hline $10-14$ & 36 & 1,4 & 17 & 2,0 & 58 & 1,1 & 111 & 1,3 \\
\hline $15-19$ & 413 & 15,7 & 131 & 15,3 & 808 & 15,8 & 1.352 & 15,8 \\
\hline $20-24$ & 517 & 19,7 & 178 & 20,7 & 1.014 & 19,9 & 1.709 & 19,9 \\
\hline $25-29$ & 393 & 15,0 & 135 & 15,7 & 872 & 17,1 & 1.400 & 16,3 \\
\hline $30-34$ & 289 & 11,0 & 69 & 8,0 & 630 & 12,4 & 988 & 11,5 \\
\hline $35-39$ & 141 & 5,4 & 51 & 5,9 & 337 & 6,6 & 529 & 6,2 \\
\hline $40-44$ & 73 & 2,8 & 17 & 2,0 & 123 & 2,4 & 213 & 2,5 \\
\hline $45-49$ & 7 & 0,3 & 5 & 0,6 & 4 & 0,1 & 16 & 0,2 \\
\hline Ignorado & 758 & 28,9 & 255 & 29,7 & 1.252 & 24,6 & 2.265 & 26,4 \\
\hline \multicolumn{9}{|c|}{ Escolaridade da mãe (anos de estudo) } \\
\hline Nenhuma & 157 & 6,0 & 37 & 4,3 & 205 & 4,0 & 399 & 4,6 \\
\hline $1-3$ & 300 & 11,4 & 96 & 11,2 & 366 & 7,2 & 762 & 8,9 \\
\hline $4-7$ & 583 & 22,2 & 178 & 20,7 & 990 & 19,4 & 1.751 & 20,4 \\
\hline $8-11$ & 487 & 18,5 & 168 & 19,6 & 1.436 & 28,2 & 2.091 & 24,4 \\
\hline 12 e mais & 76 & 2,9 & 46 & 5,4 & 439 & 8,6 & 561 & 6,5 \\
\hline Ignorado & 1.024 & 39,0 & 333 & 38,8 & 1.662 & 32,6 & 3.019 & 35,2 \\
\hline \multicolumn{9}{|l|}{ Gravidez } \\
\hline Única & 1.914 & 72,9 & 629 & 73,3 & 3.867 & 75,9 & 6.410 & 74,7 \\
\hline Múltipla & 171 & 6,5 & 63 & 7,3 & 409 & 8,0 & 643 & 7,5 \\
\hline Ignorada & 542 & 20,6 & 166 & 19,3 & 822 & 16,1 & 1.530 & 17,8 \\
\hline \multicolumn{9}{|c|}{ Gestação (semanas) } \\
\hline$<37$ & 1.136 & 43,2 & 392 & 45,7 & 2.898 & 56,8 & 4.426 & 51,6 \\
\hline $37-41$ & 688 & 26,2 & 213 & 24,8 & 1.027 & 20,1 & 1.928 & 22,5 \\
\hline$\geq 42$ & 34 & 1,3 & 8 & 0,9 & 39 & 0,8 & 81 & 0,9 \\
\hline Ignorado & 769 & 29,3 & 245 & 28,6 & 1.134 & 22,2 & 2.148 & 25,0 \\
\hline \multicolumn{9}{|l|}{ Tipo de parto } \\
\hline Vaginal & 1.227 & 46,7 & 415 & 48,4 & 2.226 & 43,7 & 3.868 & 45,1 \\
\hline Cesáreo & 827 & 31,5 & 269 & 31,4 & 1.989 & 39,0 & 3.085 & 35,9 \\
\hline Ignorado & 573 & 21,8 & 174 & 20,3 & 883 & 17,3 & 1.630 & 19,0 \\
\hline Total & 2.627 & 100,0 & 858 & 100,0 & 5.098 & 100,0 & 8.583 & 100,0 \\
\hline
\end{tabular}

SUS: Sistema Único de Saúde.

Fonte: base de dados secundária do Sistema de Informações sobre Mortalidade/Secretaria Estadual de Saúde da Paraíba, 2016.

municípios Rurais Adjacentes foram aqueles que apresentaram menor percentual desse indicador. A maior parte dos óbitos $(53,2 \%)$ aconteceu na primeira semana de vida, ou seja, na neonatal precoce e, novamente, os municípios Intermediários Adjacentes foram os que detiveram o maior percentual de óbitos neonatal precoce (54,3\%). Por outro lado, os óbitos pós-neonatal foram mais frequentes nos municípios Rurais Adjacentes (32\%) (Tabela 2). Sabe-se que os óbitos pós-neonatais são significativamente determinados por condições socioeconômicas 18 .

Entre as crianças que morreram no período do estudo, a maioria $(51,9 \%)$ tinha baixo peso ao nascer $(<2.500 \mathrm{~g})$. Os municípios Urbanos foram os que apresentaram o maior percentual de baixo peso ao nascer (56,8\%), ao passo que os Rurais Adjacentes apresentaram o maior percentual de óbitos 
infantis com não baixo peso (29,8\%). Ressalta-se, ainda, os valores "ignorados" nessa variável, aqueles não preenchidos no SIM; os municípios Rurais Adjacentes apresentaram maior frequência com 26,2\% dos óbitos, este percentual pode mascarar outro perfil da mortalidade infantil, sendo importante empreender esforço na perspectiva de sua redução.

No tocante à causa básica do óbito, identificou-se que a maioria dos óbitos infantis $(67,9 \%)$ ocorreu por causas evitáveis por intervenções do SUS. Em todos os graus de ruralidade esse porcentual ficou acima de 60\%, sendo maior nos municípios Urbanos e Intermediários Adjacentes, ambos com 69\%.

O local da ocorrência do óbito foi, majoritariamente, em hospitais (90,9\%), sendo mais frequente em municípios Urbanos (93,3\%). Por outro lado, entre os municípios Rurais Adjacentes identifica-se maior proporção de óbitos no domicílio (8\%) quando comparados com os demais municípios.

Alto percentual de óbitos infantis de mães adolescentes, aquelas situadas na faixa etária entre $10 \mathrm{e}$ 19 anos, foi observado em todos os tipos de municípios $(17,1 \%)$.

Quanto à escolaridade materna, o maior percentual de óbitos de mães analfabetas foi encontrado nos municípios Rurais (6\%) e o menor nos municípios Urbanos (4\%). Assim, os óbitos de filhos de mães de maiores níveis de escolaridade são observados nos municípios Urbanos $(8,6 \%)$, embora esta informação apresente percentual de resposta ignorada muito alto $(35,2 \%)$.

No tocante à gravidez, observou-se uma frequência aumentada de óbitos infantis nos casos de gestações múltiplas nos municípios Urbanos (8\%), comparados com os Rurais Adjacentes (6,5\%) e Intermediários Adjacentes (7,3\%). Da mesma forma que também apresentam maior proporcionalidade de óbitos entre prematuros, com menos de 37 semanas de gestação (56,8\%). Por outro lado, os municípios Rurais Adjacentes são aqueles que registraram maior percentual de óbitos infantis cuja idade gestacional ultrapassou 42 semanas (1,3\%), sugerindo dificuldade de acesso ao parto.

Do total de óbitos, $45,1 \%$ foram de crianças que passaram por parto vaginal e 35,9\% por parto cesáreo. Os municípios Intermediários Adjacentes registraram o maior percentual de partos vaginais $(48,4 \%)$ e os Urbanos o menor (43,7\%). O oposto ocorreu em relação aos partos cesáreos nos municípios Urbanos, onde identificou-se maior percentual (39\%), e nos Intermediários Adjacentes, menor $(31,4 \%)$.

\section{Discussão}

As duas tipologias rural/urbano aqui estudadas apresentam similaridades que devem ser evidenciadas. O critério fundamental considerado pelo IBGE 15 é a densidade demográfica e, além desta, a localização, a acessibilidade a centros com alto nível hierárquico em relação à rede urbana, muito semelhante à do Banco Mundial 16.

O diferencial da tipologia do IBGE foi o tratamento dado à densidade demográfica que metodologicamente mostrou-se mais adequado à dinâmica espacial brasileira. Sendo esse tratamento metodológico uma das explicações para a melhor resposta à comparação aplicada neste trabalho.

A tipologia do IBGE mostrou-se útil na caracterização do perfil epidemiológico do óbito infantil. Produzindo resultados coerentes com outros estudos, sobretudo, quanto ao baixo peso ao nascer e à prematuridade 3,4,25. Representa um caminho viável para o monitoramento constante da mortalidade infantil, nos municípios Rurais Adjacentes, no que tange ao cumprimento dos ODS.

Obviamente, o exercício metodológico descrito neste artigo pressupõe a necessidade de testes e validações futuros buscando o seu aperfeiçoamento. Entretanto, mesmo com o risco da "simplificação da realidade" 15 imposta pela tipologia adotada, o estudo traz a possibilidade de identificar fragilidades no âmbito municipal na perspectiva da ruralidade, semelhante ao encontrado por Vianna et al. ${ }^{4} \mathrm{em}$ outro nível de desagregação, que foi a análise dos dados dos óbitos infantis por macrorregionais de saúde. Seus achados identificaram fragilidades nas áreas estudadas que puderam ser priorizadas pelos gestores de saúde, contribuindo para o monitoramento e avaliação da mortalidade infantil.

A utilização de dados secundários, do SIM, propiciou a implantação do presente estudo, contribuindo para o entendimento da situação de saúde dos espaços rurais. A variável "município de residência da mãe" possibilitou relacionar o óbito infantil à tipologia do IBGE, resultando na caracterização do óbito infantil nos municípios rurais. Essa mesma variável está presente no Sistema de Informações sobre Nascidos Vivos (SINASC), com isto torna-se possível desvelar outras dimensões 
das desigualdades vivenciadas nos municípios rurais - como exemplo: taxa de partos cesáreos no SUS e proporção de nascidos vivos de mães com sete ou mais consultas pré-natal - contribuindo para a redução da invisibilidade da situação de saúde da população rural.

Bases de dados demográficas, SIM e SINASC, dos sistemas de informação em saúde do Brasil, são as melhores fontes de informação secundária disponíveis, e importantes estudos são realizados utilizando estes mesmos dados 26,27,28. Nesse sentido, é importante reforçar a necessidade da melhoria da coleta de informações pelos serviços de saúde e, consequentemente, buscar a redução de respostas ignoradas nos bancos de dados do SIM.

O comportamento declinante do coeficiente de mortalidade infantil no período estudado foi de $34,32 \%$, semelhante ao encontrado em outros trabalhos que identificam esta tendência de declínio 29,30,31, exceto entre os anos de 2015 e 2016. Esse comportamento é observado em todo o país, independentemente do porte populacional dos municípios ${ }^{32}$, ou seja, contemplando também os municípios Rurais Adjacentes. Contudo, ao observar o número total de óbitos infantis e de nascidos vivos, apenas em 2016 percebe-se o aumento do primeiro e a retração do segundo. A Associação Brasileira de Saúde Coletiva (Abrasco) 33 alertou sobre essa mudança observada em 2016. No Brasil, de 2015 para 2016 o aumento foi de 12,4 para 12,7/mil nascidos vivos. Esse aumento foi observado em todas as regiões do país, exceto na Região Sul, e pode estar associado à crise econômica, ao ajuste fiscal e aos cortes no orçamento da saúde 33 .

A redução da TMI depende de melhorias nas condições de vida e na implantação de políticas públicas de saúde, exigindo comprometimento e desempenho dos gestores públicos nas três esferas de governo. A ampliação da atenção primária em saúde e a implantação de outros programas com foco na melhoria da saúde e da nutrição infantil repercutiram, positivamente, nesse indicador no caso do Brasil 26,30.

A maior participação do componente neonatal vem sendo evidenciada em outros estudos 1,4,26. Esse resultado explica o esforço de organismos internacionais acerca da redução desse componente da mortalidade infantil 6 . No período estudado, o percentual de mortalidade precoce na Paraíba foi de 53,2\% e nos municípios Rurais Adjacentes, 52,4\%.

Sabe-se que, assim como a prematuridade, o baixo peso ao nascer é um fator associado ao óbito infantil. Um estudo realizado em Caxias do Sul (Rio Grande do Sul) 1 detectou que crianças com menos de $2.500 \mathrm{~g}$ ao nascer apresentam seis vezes mais riscos de morrer no período neonatal precoce do que crianças com peso maior.

Embora nossos achados tenham apresentado que 51,9\% dos óbitos infantis tinham menos de $2.500 \mathrm{~g}$ ao nascer, este resultado requer cautela. Outros trabalhos apresentaram percentuais bem maiores que esse 4,26 , mais especificamente, entre $60 \%$ e $80 \%$. Esse baixo percentual comparado aos outros estudos pode estar relacionado ao não preenchimento de variáveis que assumiram valor "ignorado".

Quanto a tal questão, evidencia-se que, exceto pela variável raça/cor, os campos "ignorados" foram mais frequentes nos municípios Rurais Adjacentes do que nos Urbanos. Diante disso, cabe reforçar a necessidade da melhoria da coleta de informações pelos serviços de saúde, principalmente nos municípios Rurais Adjacentes, buscando a redução de respostas ignoradas no banco de dados do SIM. O não preenchimento de variáveis que caracterizam o perfil da criança e da mãe indica a necessidade de capacitação/sensibilização dos profissionais de saúde envolvidos com estes sistemas vitais e ainda pressupõe fragilidades da vigilância do óbito infantil na Paraíba.

Sobre a incompletude dos dados, um estudo realizado em Recife (Pernambuco) evidenciou a contribuição da vigilância do óbito para o aprimoramento das estatísticas vitais. Entre seus achados, os autores identificaram que a média de incompletude das variáveis dos óbitos menores de um ano era de $11,1 \%$ antes da investigação, e após a investigação verificou-se a inexistência de variáveis em branco ou ignoradas 34 .

Todas as análises consideraram os municípios como unidade amostral, sendo, portanto, uma análise agregada dos dados de mortalidade infantil. Esse fato, inerente à metodologia dos estudos ecológicos, possibilita a ocorrência de um erro comum conhecido como falácia ecológica 18, ou seja, mesmo em um município de característica rural, o óbito infantil pode ter acontecido com uma mãe residente na área urbana do município, ou vice-versa. Essa limitação somente pode ser resolvida com a classificação devida dos domicílios e a incorporação desta informação na declaração de óbito da 
criança. Outra limitação refere-se ao cálculo do tempo de viagem utilizado para compor a tipologia do Banco Mundial que foi dado pela distância euclidiana, desconsiderando a via de transporte real.

Entretanto, para efeito deste trabalho, considera-se que a nova classificação do IBGE significa um importante avanço na classificação dos municípios, e os fatores relacionados ao óbito infantil em cada município, por sua vez, devem ser o resultante das interações sociais, econômicas, de oferta de serviços, acessibilidade e a relação entre as diferentes formas de morar e de viver das famílias nas diferentes áreas do município. Considerando a complexidade dessa relação, deparamos com fatores diferentes associados ao óbito infantil, levando-se em conta a tipologia de níveis de ruralidade adotada.

\section{Colaboradores}

R. A. S. Soares participou da concepção do trabalho e da aquisição, análise e interpretação dos dados; redigiu o texto e revisou-o criticamente. R. M. Moraes e R. P. T. Vianna participaram da concepção do trabalho, revisaram criticamente o texto e aprovaram a versão final.

\section{Informações adicionais}

ORCID: Rackynelly Alves Sarmento Soares ( 00000002-6759-4398); Ronei Marcos de Moraes (00000001-8436-8950); Rodrigo Pinheiro de Toledo Vianna (0000-0002-5358-1967).

\section{Referências}

1. Bozzetti MC, Araújo BF, Tanaka ACA. Early neonatal mortality in Caxias do Sul: a cohort study. J Pediatr (Rio J.) 2000; 76:200-6.

2. Tavares LT, Albergaria TFS, Guimarães MAP, Pedreira RBS, Pinto-Junior EP. Mortalidade infantil por causas evitáveis na Bahia, 20002012. RECIIS (Online) 2016; 10(3). https:// www.reciis.icict.fiocruz.br/index.php/reciis/ article/view/1044.

3. Departamento de Análise de Situação de Saúde, Secretaria de Vigilância em Saúde, Ministério da Saúde. Saúde Brasil 2011: uma análise da situação de saúde e a vigilância da saúde da mulher. Brasília: Editora do Ministério da Saúde; 2012.

4. Vianna RCXF, Freire MHS, Carvalho D, Migotto MT. Perfil da mortalidade infantil nas Macrorregionais de Saúde de um estado do Sul do Brasil, no triênio 2012-2014. Revista de Saúde Pública do Paraná 2016; 17:32-40.

5. United Nations Children's Fund. Child mortality report 2015. http://www.who.int/ma ternal_child_adolescent/documents/levels_ trends_child_mortality_2015/en/ (acessado em 12/Out/2017).

6. United Nations. 2030 agenda for sustainable development. https://www.cepal.org/en/ news/2030-agenda-sustainable-development (acessado em 12/Dez/2017).

7. Atlas Brasil. Atlas de Desenvolvimento Humano no Brasil 2013. http://atlasbrasil.org. br/2013/pt/ (acessado em 16/Nov/2017).

8. Departamento de Ações Programáticas Estratégicas, Secretaria de Atenção à Saúde, Ministério da Saúde. Pacto pela redução da mortalidade infantil no Nordeste e Amazônia Legal: 2009-2010. Brasília: Ministério da Saúde; 2010.

9. Guerrero AFH, Silva DO, Toledo LM, Guerrero JCH, Teixeira P. Mortalidade infantil em remanescentes de quilombos do Município de Santarém - Pará, Brasil. Saúde Soc 2007; 16:103-10. 
10. Secretaria de Gestão Estratégica e Participativa, Ministério da Saúde. Política Nacional de Saúde Integral das Populações do Campo e da Floresta. Brasília: Editora do Ministério da Saúde; 2013.

11. Soares RAS, Moraes RM, Pessoa VM, Carneiro FF, Vianna RPT. A invisibilidade da população do campo, floresta e das águas no Brasil: desafio para os sistemas de informações em saúde. In: Carneiro FF, Pessoa VM, Teixeira ACA, organizadores. Campo, floresta e águas: práticas e saberes em saúde. Brasília: Editora da UnB; 2017. p. 106-24.

12. Programa das Nações Unidas para o Desenvolvimento. Desenvolvimento humano para além das médias: 2017. Brasília: Programa das $\mathrm{Na}$ ções Unidas para o Desenvolvimento/Instituto de Pesquisa Econômica Aplicada/Fundação João Pinheiro; 2017.

13. Miranda C, Silva H. Concepções da ruralidade contemporânea: as singularidades brasileiras. Brasília: Instituto Interamericano de Cooperação para a Agricultura; 2013.

14. Instituto Paranaense de Desenvolvimento Econômico e Social. Redefinição dos conceitos de urbano e rural. http://www.ipardes.gov. br/biblioteca/docs/redefinicao_urbano_ru ral_12_83.pdf (acessado em 26/Jul/2015).

15. Instituto Brasileiro de Geografia e Estatística. Classificação e caracterização dos espaços rurais e urbanos do Brasil: uma primeira aproximação. Rio de Janeiro: Instituto Brasileiro de Geografia e Estatística; 2017.

16. Ferranti D, Perry GE, Foster W, Lederman D, Valdés A. Beyond the city: the rural contribution to development. Washington DC: World Bank; 2005.

17. Unglert CVS, Rosenburg CP, Junqueira CB. Acesso aos serviços de saúde: uma abordagem de geografia em saúde pública. Rev Saúde Pública 1987; 21:439-46.

18. Rouquayrol MZ, Almeida Filho N. Epidemiologia \& saúde. 6a Ed. Rio de Janeiro: Medsi; 2003.

19. Malta DC, Duarte EC, Almeida MF, Dias MAS, Morais Neto OL, Moura L, et al. Lista de causas de mortes evitáveis por intervenções do Sistema Único de Saúde do Brasil. Epidemiol Serv Saúde 2007; 16:233-44.

20. Brasil. Lei no 9.503 , de 23 de setembro de 1997. Institui o Código de Trânsito Brasileiro. Diário Oficial da União 1997; 24 set.

21. Sorokin PA, Zimmerman CC, Galpin CJ. Diferenças fundamentais entre o mundo rural e o urbano. In: Martins JS, organizador. Introdução crítica à sociologia rural. São Paulo: Editora Hucitec; 1981. p. 198-224.

22. Soares RAS, Moraes RM, Vianna RPT, Pessoa VM, Carneiro FF. Determinantes socioambientais e saúde: o Brasil rural versus o Brasil urbano. Tempus (Brasília) 2015; 9:221-35.
23. Silva CF, Rodrigues CT, Monteiro MVB. Uso de regras de associação para descoberta de conhecimento na produtividade de açaí no Estado do Amapá. In: Anais do V Congresso Sul Brasileiro de Computação. http://periodicos. unesc.net/sulcomp/issue/view/25 (acessado em 05/Mar/2018).

24. Landis J, Koch GG. The measurements of agreement for categorical data. Biometrics 1977; 33:159-79.

25. Díaz AA, Bacallao Gallestey J, Vargas-Machuca R, Aguilar Velarde R. Desarrollo infantil en zonas pobres de Perú. Rev Panam Salud Pública 2017; 41:e71.

26. Departamento de Análise de Situação de Saúde, Secretaria de Vigilância em Saúde, Ministério da Saúde. Saúde Brasil 2012. Brasília: Editora do Ministério da Saúde; 2012.

27. Soares ES, Menezes GMS. Fatores associados à mortalidade neonatal precoce: análise de situação no nível local. Epidemiol Serv Saúde 2010; 19:51-60.

28. Frias PG, Szwarcwald CL, Morais Neto OL, Leal MC, Cortez-Escalante JJ, Souza Junior PRB, et al. Utilização das informações vitais para a estimação de indicadores de mortalidade no Brasil: da busca ativa de eventos ao desenvolvimento de métodos. Cad Saúde Pública 2017; 33:e00206015.

29. Organización Panamericana de la Salud. Salud en las Américas+, edición del 2017. Resumen: panorama regional y perfiles de país. Washington DC: Organización Panamericana de la Salud; 2017. (Publicación Científica y Técnica, 642).

30. Victora CG, Aquino EML, Leal MC, Monteiro CA, Barros FC, Szwarcwald CL. Maternal and child health in Brazil: progress and challenges. Lancet 2011; 377:1863-76.

31. Malta DC, Duarte EC, Escalante JJC, Almeida MF, Sardinha LMV, Macário EM, et al. Mortes evitáveis em menores de um ano, Brasil, 1997 a 2006: contribuições para a avaliação de desempenho do Sistema Único de Saúde. Cad Saúde Pública 2010; 26:481-91.

32. Departamento de Análise de Situação em Saúde, Secretaria de Vigilância em Saúde, Ministério da Saúde. Saúde Brasil 2010: uma análise da situação de saúde e de evidências selecionadas de impacto de ações de vigilância em saúde. Brasília: Ministério da Saúde; 2011.

33. Reis V. Especial Abrasco sobre o aumento da mortalidade infantil e materna no Brasil. https://www.abrasco.org.br/site/outras-no ticias/institucional/especial-abrasco-sobre-oaumento-da-mortalidade-infantil-e-mater na-no-brasil/36777/ (acessado em Out/2018).

34. Oliveira CM, Bonfim CV, Guimarães MJ, Frias PG, Medeiros ZM. Mortalidade infantil: tendência temporal e contribuição da vigilância do óbito. Acta Paul Enferm 2016; 29:282-90. 


\section{Abstract}

The study aimed to compare two concepts of rurality, one proposed by the Brazilian Institute of Geography and Statistics (IBGE) and the other by the World Bank, to determine which is better fitted to the territory's reality, besides analyzing the infant mortality profile of rural municipalities (counties) in the state of Paraíba, Brazil, according to the best criterion for rurality. This was an observational epidemiological study conducted in the state of Paraiba. The method for analyzing rural/ urban typologies was based on data mining, using the Apriori algorithm of association. Infant mortality was analyzed with descriptive statistics. The data were obtained from the Mortality Information System of the Brazilian Ministry of Health, from 2007 to 2016, and municipal indicators were from IBGE. The World Bank definition of rurality showed kappa $=0.337$, compared to the IBGE definition, with kappa $=0.616$. Among the $223 \mathrm{mu}$ nicipalities that were analyzed, the World Bank classified 130 (65.66\%) correctly, and the IBGE 183 (82.06\%). The predominant epidemiological profile of infant mortality in rural municipalities in Paraiba state was male gender (57.4\%), brown skin color (61.1\%), age from 0 to 7 days (52.4\%), low birthweight (44\%), and gestational age less than 37 weeks (43.2\%). Underlying cause of death was classified as avoidable death via interventions by the Brazilian Unified National Health System (65.2\%). The urban/rural typology adopted by the IBGE was better than the World Bank at classifying the municipalities in Paraiba state. This classification allowed studying the infant mortality profile in rural municipalities, which was similar to the overall profile, except for maternal schooling.

Infant Mortality; Vital Statistics; Rural Health; Social Determinants of Health; Health Equity

\section{Resumen}

El objetivo del estudio fue comparar dos propuestas de ruralidad, la del Instituto Brasileño de Geografía y Estadística (IBGE) y la del Banco Mundial, respondiendo cuál de ellas se adecua mejor a la realidad en el territorio, así como conocer el perfil de mortalidad infantil en municipios rurales de Paraíba, Brasil, según el criterio mejor evaluado de ruralidad. Se trata de un estudio epidemiológico observacional, realizado en el estado de Paraíba. El método de análisis de las tipologías rural/urbana se basó en la mineración de datos, a partir del algoritmo de asociación Apriori. Para el análisis del óbito infantil, se utilizó la estadística descriptiva. Se consideraron los datos del Sistema de Informaciones de Mortalidad del Ministerio de Salud de Brasil, durante el período de 2007 a 2016, así como indicadores municipales del IBGE. La tipología de ruralidad del Banco Mundial presentó kappa $=0,337$ y la del IBGE kappa $=0,616$. Entre los 223 municipios analizados, la tipología del Banco Mundial clasificó correctamente 130 (65,66\%) y la del IBGE 183 (82,06\%). El perfil epidemiológico predominante en los municipios rurales paraibanos fue: sexo masculino $(57,4 \%)$, color pardo (61,1\%), edad entre 0 y 7 días $(52,4 \%)$, con bajo peso al nacer (44\%), edad gestacional menor de 37 semanas $(43,2 \%)$. La causa básica de la muerte fue clasificada como muerte evitable por intervenciones del Sistema Único de Salud $(65,2 \%)$. La tipología urbano/rural, presentada por el IBGE, clasificó mejor los municipios paraibanos que la del Banco Mundial. Esta clasificación posibilitó el estudio del perfil del óbito infantil en los municipios rurales, a través del cual se verificó, excepto por la escolaridad de la madre, similitud con el perfil general.

Mortalidad Infantil; Estadísticas Vitales; Salud Rural; Determinantes Sociales de la Salud; Equidad en Salud
Recebido em 05/Abr/2018

Versão final reapresentada em 02/Ago/2019

Aprovado em 31/Jan/2020 